\section{P92 Needs Assessment for a Mobile Text Messaging Intervention for SNAP-Eligible Adults}

Brigette Herron,PhD, Brig07@uga.edu, University of Georgia, 115 DW Brooks Dr, Athens, GA, 30602; Danielle Anthony, $B A$, University of Georgia; Darci Bell, RDN, LD, University of Georgia; Edda Cotto-Rivera, MPH, CHES, University of Georgia; Jung Sun Lee, PhD, RDN, University of Georgia

Background: Mobile text messaging intervention is a promising method for improving health promotion and behavior change. Little is known about feasibility and strategies for text messaging interventions targeted to low-income adults.

Objective: The objective of this study was to explore opinions about feasibility, tone, and content for the development of a texting campaign for Supplemental Nutrition Assistance Program Education (SNAP-Ed) eligible adults to encourage drinking more water and drinking less sugarsweetened beverages (SSBs).

Study Design, Setting, Participants: A qualitative research design was used to elicit the opinions of SNAP-Ed eligible individuals living in the state of Georgia. Using a convenience sample, 13 former SNAP-Ed direct education participants (84.6\% female, 69.2\% African American) were recruited by text message and email and participated in 4 focus groups and 1 in-depth interview through Zoom. Interviews focused on participants' texting behavior, current beverage consumption, and proposed content of text messages.

Measurable Outcome/ Analysis: Focus group interviews were digitally recorded, transcribed verbatim, and coded to establish emergent themes using constant comparative method and Atlas.ti.

Results: Participants indicated text messaging would be an affordable, desirable, and comfortable way to receive information about nutrition and health. Participants expressed a desire to increase water consumption for themselves due to existing health concerns and wanted to reduce the number of SSBs they drank per day, despite currently drinking SSBs daily. The price and taste of water and unsweetened beverages were reported as the most common barriers to making healthier drink choices. Participants preferred content relevant to their lives (including health issues and abilities), and desired opportunities to build upon their previous knowledge with action-oriented, interactive, and engaging text messages.

Conclusion: Text messaging is a feasible method to provide nutrition education to SNAP-Ed eligible adults. The findings from this study will inform and guide a new University of Georgia SNAP-Ed text messaging intervention Funding: Supplemental Nutrition Assistance Program Education.

\section{P93 Precision Behavioral Nutrition: Development of the NutriPCP Inference Engine for Data-driven Diet Goals in Primary Care}

Madalyn Rosenthal, BS, madalyn.rosenthal@austin.utexas. edu, The University of Texas at Austin, Department of Nutritional Sciences, 200 W 24th St, Austin, TX, 78705; Dagny Larson, $B S, L D, R D N, C S R$, The University of Texas at
Austin, Department of Nutritional Sciences; Jacqueline Henning, BS, The University of Texas at Austin, Department of Nutritional Sciences; Eesha Nayak, The University of Texas at Austin, Cockrell School of Engineering; Gracia Dala, BS, RD, The University of Texas at Austin, Department of Nutritional Sciences; Krystal Martinez, $B S, R D, L D$, The University of Texas at Austin, Department of Nutritional Sciences; Brandon A. Altillo, $M P H, M D$, Lone Star Circle of Care; The University of Texas at Austin, Dell Medical School, Department of Internal Medicine; Department of Population Health; Department of Pediatrics; Marissa Burgermaster, PhD, MA, MEEd, The University of Texas at Austin, Department of Nutritional Sciences; Dell Medical School, Department of Population Health

Objective: To develop a computational system that uses dietary recall data to prioritize behavioral goals to facilitate efficient, personalized collaborative goal-setting in primary care.

Use of Theory or Research: The Chronic Care Model posits that synergy between the healthcare system and patient self-management will improve chronic disease outcomes. Thus, improving how diet is addressed in primary care could augment the benefit of dietary self-management. Collaborative goal-setting with primary care providers (PCPs) can facilitate patient behavior change. However, PCPs lack time and training to set effective diet goals with patients. NutriPCP aims to address this gap by presenting PCPs with a set of evidence-based goals prioritized using patient data.

Target Audience: PCPs

Program Description: NutriPCP uses ASA24 diet recall data to compute patient status for each of 9 previously developed, MyPlate-based goals (eg, "Make half my grains whole"). NutriPCP's inference engine consists of Python rule statements that synthesize a patient's data and compare it to evidence-based targets for nutrient consumption personalized for patient characteristics (eg, kcal intake/sex/age). PCPs are then presented with a list of the patient's status for each goal prioritized by degree of improvement needed.

Evaluation Methods: We tested our inference engine with test data $(\mathrm{n}=12)$, and our team of nutrition, technology, and clinical experts validated the output. We used NHANES data to establish reasonable population-wide estimates.

Results: Testing revealed challenges for goal prioritization because datasets reflected consumption far from evidence-based targets. Therefore, we created standardized ranges to improve variability for relative ranking across goals. For goals with upper and lower limits (eg, "Reduce portion size") we added warnings for inadequate intake.

Conclusion: We demonstrated that computational rules can automatically process recall data into prioritized behavioral goals. To our knowledge, this is the first system that personalizes MyPlate recommendations based on an individual's data. This has implications for nutrition education in primary care. Future research will examine implementation feasibility for PCPs and patients.

Funding: Agency for Healthcare Research and Quality (AHRQ) R 21 HS027660 Burgermaster, PI. 